CERN-TH.6941/93

hep-ph/9310286

UICHEP-TH/93-15

\title{
The Decay Rate Asymmetry of the Top Quark
}

\author{
Bohdan Grządkowskił \\ Wai-Yee Keung? \\ Theory Division, CERN, CH-1211 Geneva 23, Switzerland
}

\begin{abstract}
The asymmetries $\mathcal{A}_{k} \equiv\left[\Gamma\left(t \rightarrow W^{+} d_{k}\right)-\Gamma\left(\bar{t} \rightarrow W^{-} \bar{d}_{k}\right)\right] /\left[\Gamma\left(t \rightarrow W^{+} d_{k}\right)+\Gamma\left(\bar{t} \rightarrow W^{-} \bar{d}_{k}\right)\right]$ in the partial widths of the top quark decays are discussed within the Standard Model (SM), the Two-Higgs-Doublet Model (2HDM) and supersymmetric extensions of the SM (SSM). The leading contributions to these asymmetries in the SM and in the 2HDM are induced by the up-type quark self-energy diagrams and are found to be very small. However, in the SSM, the asymmetry $\mathcal{A}_{b}$ can be substantial, $\mathcal{O}\left(\alpha_{Q C D}\right)$, provided the CP-violating phase of gluino-top-stop couplings is not suppressed. Within the $\operatorname{SSM} \mathcal{A}_{b}$ is generated by the vertex corrections.
\end{abstract}

CERN-TH.6941/93

July 1993

\footnotetext{
${ }^{1}$ E-mail: bohdang@cernvm.

Address after 1 October 1993: Institute for Theoretical Physics, University of Warsaw, Hoża 69, PL-00681 Warsaw, Poland.

${ }^{2}$ E-mail: keung@fnal .

Address after 1 August 1993: Physics Department, M/C 273 University of Illinois at Chicago, IL 606077059 , USA.
} 


\section{Introduction}

A very heavy top quark $t$ can offer a few relevant advantages for the study of CP violation:

- If $m_{t}>130 \mathrm{GeV}$, it would decay before it can form a bound state [1]; therefore the perturbative description is much more reliable.

- For the same reason, the spin information of the top quark would not be diluted by hadronization, so that the spin effects can provide a very useful tool in searching for $\mathrm{CP}$ violation.

- Again, because of the large mass of the top quark, its properties are sensitive to interactions mediated by Higgs bosons [2].

- The Kobayashi-Maskawa [3] mechanism of CP violation is strongly suppressed for the top quark since its mixing with other generations is very weak; therefore it is sensitive to non-conventional sources of $\mathrm{CP}$ violation.

Discussion of heavy-quark rate asymmetries has been initiated by the pioneering article by Bander, Silverman and Soni in Ref. [4], which was devoted to the bottom-quark decays. The same mechanism of the rate-asymmetry generation was then improved [5] and eventually applied to the top quark. The top-quark decay-rate asymmetry has been discussed in the literature [6], usually for cases of 3-body decays, which make the problem much more involved. Here, we will concentrate on dominant 2-body decays of the top quark: $t \rightarrow W^{+} d_{k}$. Other consequences of $\mathrm{CP}$ violation due to the top quark spin effects can be found in refs. [7]-[1].

\section{$2 \quad \mathrm{SM}$ and $2 \mathrm{HDM}$}

Let us denote the contribution from $d_{i}$ internal quark to the mixed $t-u_{j}$ quark self-energy diagram by

$$
i\left\{\not p_{t}\left[P_{L} \Sigma_{L}\left(m_{i}^{2}\right)+P_{R} \Sigma_{R}\left(m_{i}^{2}\right)\right]+P_{L} m_{j} \Sigma_{L}^{S}\left(m_{i}^{2}\right)+P_{R} m_{t} \Sigma_{R}^{S}\left(m_{i}^{2}\right)\right\}
$$

where $P_{L / R}$ are the chiral projection operators, $p_{t}$ is the incoming top-quark momentum, $m_{t}$ denotes the top-quark mass, $p_{t}^{2}=m_{t}^{2}$, and $m_{j}$ is the mass of the outgoing $u_{j}$ quark. Hereafter we assume that $V_{i j}$-elements of the Kobayashi-Maskawa (KM) matrix [3] are factorized out and do not enter into $\Sigma$ 's. The analogous expression can be written for the incoming antitop of momentum $p_{\bar{t}}$;

$$
i\left\{p_{\bar{t}}\left[P_{L} \bar{\Sigma}_{L}\left(m_{i}^{2}\right)+P_{R} \bar{\Sigma}_{R}\left(m_{i}^{2}\right)\right]+P_{L} m_{t} \bar{\Sigma}_{L}^{S}\left(m_{i}^{2}\right)+P_{R} m_{j} \bar{\Sigma}_{R}^{S}\left(m_{i}^{2}\right)\right\}
$$

It is easy to see that the following relations hold:

$$
\Sigma_{L / R}=-\bar{\Sigma}_{L / R} \text { and } \Sigma_{L / R}^{S}=\bar{\Sigma}_{R / L}^{S}
$$

For the tree-level amplitude we use the following notation:

$$
A_{1}=V_{t k}^{*} \hat{A}_{1}, \quad \hat{A}_{1}=-\frac{i g}{\sqrt{2}} \bar{u}\left(p_{k}\right) \gamma_{\mu} P_{L} u\left(p_{t}\right) \epsilon^{\mu}
$$


where $\epsilon^{\mu}$ is the $W$-boson polarization vector. The diagram in Fig. 1 with the self-energy inserted before the decay vertex produces a 1-loop amplitude for the top-quark decay:

$$
A_{2}=-\hat{A}_{1} \sum_{i j} V_{t i}^{*} V_{j i} V_{j k}^{*} \frac{m_{t}^{2}}{m_{t}^{2}-m_{j}^{2}} \Sigma\left(m_{i}^{2}\right),
$$

where $i$ denotes the internal down-type quark flavour and

$$
\Sigma\left(m_{i}^{2}\right) \equiv \Sigma_{L}\left(m_{i}^{2}\right)+\Sigma_{R}^{S}\left(m_{i}^{2}\right)+\frac{m_{j}}{m_{t}} \Sigma_{R}\left(m_{i}^{2}\right)+\frac{m_{j}^{2}}{m_{t}^{2}} \Sigma_{L}^{S}\left(m_{i}^{2}\right) .
$$

A similar expression can be obtained for the antitop decay. The rate difference between

Figure 1: The self-energy induced contribution to $\mathcal{A}_{k}$. The vertical dashed line denotes the absorptive part of the corresponding integral.

top and antitop decay is given by the interference of the tree-level and 1-loop amplitudes:

$$
2 m_{t}(\Gamma-\bar{\Gamma})=2 \int d \Phi\left(W^{+} d_{k}\right)\left[\operatorname{Re}\left(A_{1} A_{2}^{*}\right)-\operatorname{Re}\left(\bar{A}_{1} \bar{A}_{2}^{*}\right)\right]
$$

where $\Gamma \equiv \Gamma\left(t \rightarrow W^{+} d_{k}\right), \bar{\Gamma} \equiv \Gamma\left(\bar{t} \rightarrow W^{-} \bar{d}_{k}\right), \bar{A}_{1}$ and $\bar{A}_{2}$ stands for the tree and 1-loop induced amplitudes for the antitop decay. For the phase-space element $d \Phi(\cdots)$, we use:

$$
d \Phi=(2 \pi)^{4} \prod_{i=1}^{n_{f}} \frac{d^{3} p_{i}}{(2 \pi)^{3} 2 E_{i}} \delta^{(4)}\left(P_{i n}-\sum_{i=1}^{n_{f}} p_{i}\right),
$$

As is usual for rate asymmetries, since CP-violating couplings enter with opposite signs for the top and the antitop decays, the asymmetry can be non-zero if there is an absorptive part of the 1-loop contribution to the amplitude:

$$
\mathcal{A}_{k} \equiv \frac{\Gamma-\bar{\Gamma}}{\Gamma+\bar{\Gamma}}=-2 \sum_{i j} \frac{\operatorname{Im}\left(V_{t k}^{*} V_{t i} V_{j i}^{*} V_{j k}\right)}{\left|V_{t k}\right|^{2}} \frac{m_{t}^{2}}{m_{t}^{2}-m_{j}^{2}} \operatorname{Im}\left[\Sigma\left(m_{i}^{2}\right)\right],
$$

where the relations of Eq. (3) have been adopted. The above formula has a few relevant features. Since in the SM there is only one CP-violating parameter, our asymmetry must be proportional to it; in fact all non-zero values of $\operatorname{Im}\left(V_{t k}^{*} V_{t i} V_{j i}^{*} V_{j k}\right)$ satisfy:

$$
\operatorname{Im}\left(V_{t k}^{*} V_{t i} V_{j i}^{*} V_{j k}\right)= \pm J
$$

The $J$ factor, which is invariant under reparametrization [12], can be rewritten in terms of the Wolfenstein parameters [13] of the KM matrix:

$$
J=A^{2} \lambda^{6} \eta
$$

where $A \simeq 1, \lambda=\sin \theta_{C}=0.22$, and $\eta$ is a CP-violating parameter of the KM matrix. Because of the unitarity of the KM matrix, it is clear that non-zero contributions to the sum in Eq. (9) appear only if $i \neq k$ and $j \neq t$. This supports the common belief [5] that rescattering of the initial (or final) state does not contribute to rate asymmetries. The 
last important remark is that the asymmetry is doubly GIM-suppressed; first because the loop is summed over $i$ and second, because it is also summed over $j$. Therefore we expect

$$
\mathcal{A}_{k} \sim \lambda^{6} \frac{\left(m_{u_{l}}^{2}-m_{u_{m}}^{2}\right)}{m_{W}^{2}} \frac{\left(m_{d_{n}}^{2}-m_{d_{o}}^{2}\right)}{m_{W}^{2}}
$$

This turns out to be a very small number. The asymmetry within the SM may only be of an academic interest; however it is instructive to note what the suppression factors are, in order to overcome them within some modifications of the KM mechanism of CP violation. It also illustrates many generic features of $\mathrm{CP}$ violation. For completeness we present here the result for $\mathcal{A}_{k}$ calculated within the $2 \mathrm{HDM}$ under the assumption that decays $t \rightarrow H^{+} d_{k}$ are kinematically allowed. The relevant contributions to the amplitudes are given by similar diagrams as in Fig. 1, with the internal $W$ line replaced by the charged Higgs boson line. We do not consider the possibility that $\mathrm{CP}$ is violated through interactions of neutral Higgs bosons and we keep only contributions growing with $\tan ^{2} \beta \equiv\left(v_{2} / v_{1}\right)^{2}$ :

$$
\mathcal{A}_{k}=-\frac{\alpha}{8 \sin ^{2} \theta_{W}}\left[f_{W}(y)+\tan ^{2} \beta f_{H}(x, y)\right] \sum_{i j} \frac{\operatorname{Im}\left(V_{t k}^{*} V_{t i} V_{j i}^{*} V_{j k}\right)}{\left|V_{t k}\right|^{2}} \frac{m_{i}^{2}}{m_{W}^{2}} \frac{m_{j}^{2}}{m_{W}^{2}},
$$

where the leading terms in the expansion $m_{i, j}^{2} / m_{W}^{2}$ have been kept. The $f_{W}$ and $f_{H}$ are functions originating from imaginary parts of self-energies involving $W$ and $H$ exchange, respectively; they are given by:

$$
\begin{aligned}
f_{W}(y)=-3\left(1+y^{4}\right) / y^{6} & , \quad f_{H}(x, y)=\left(1-x^{2}\right)^{2} /\left(x^{4} y^{2}\right) \\
y \equiv m_{t} / m_{W} & , \quad x \equiv m_{t} / m_{H}
\end{aligned}
$$

Eventually, assuming $A=1$, we can write the following expression:

$$
\mathcal{A}_{k}=-\frac{\alpha \eta}{8 \sin ^{2} \theta_{W}} \frac{m_{b}^{2} m_{c}^{2}}{m_{W}^{4}}\left[f_{W}(y)+\tan ^{2} \beta f_{H}(x, y)\right]\left\{\begin{array}{cl}
-\left[(1-\rho)^{2}+\eta^{2}\right]^{-1} & \text { for } k=d \\
\lambda^{2} & \text { for } k=s \\
-\lambda^{6} m_{s}^{2} / m_{b}^{2} & \text { for } k=b .
\end{array}\right.
$$

From the above we see that $\mathcal{A}_{k}$ can be, within the SM, at best of the order of $10^{-9}$, and even that tiny number can only be reached for the rarest mode: $t \rightarrow W^{+} d$. Since $\tan \beta$ is limited from above by the measurement [14 of the branching ratio for $b \rightarrow c \tau \nu_{\tau}$ $\left(\tan \beta<0.54\left[m_{H} / 1 \mathrm{GeV}\right]\right)$ we can conclude that even within $2 \mathrm{HDM}$ we are not able to enhance the rate asymmetry. However, if we admit more than three generations of

quarks $\mathcal{A}_{k}$ may become much bigger, since in that case we can overcome both sources of suppression: no $\lambda^{6}$ factor and a new $t^{\prime}$ quark can have a comparable mass as $m_{t}$ so that the suppression due to the $u_{j}$ propagator is absent.

\section{Supersymmetric Standard Models}

In this section we will restrict ourselves to the dominant top-quark decay mode: $t \rightarrow W^{+} b$ and effectively neglect any flavour mixing. Supersymmetric versions [15] of the SM offer 
new sources of $\mathrm{CP}$ violation. We write down the $\mathrm{CP}$-violating interaction that is relevant for us:

$$
\begin{aligned}
\mathcal{L}= & i \sqrt{ } 2 g_{s}\left[\tilde{t}_{L}^{*} T^{a}\left(\bar{\lambda}^{a} t_{L}\right)+\tilde{t}_{R}^{*} T^{a}\left(\bar{\lambda}^{a} t_{R}\right)\right]+(t \leftrightarrow b) \\
& -(i / \sqrt{2}) V_{t b} \tilde{b}_{L}^{\dagger} \stackrel{\leftrightarrow}{\partial}_{\mu} \tilde{t}_{L} W^{-\mu}+\text { h.c. },
\end{aligned}
$$

where $g_{s}$ is the QCD coupling constant. For our purpose the most relevant new source of $\mathrm{CP}$ violation, which appears in the SSM, would be the phase in the $\tilde{t}_{L}-\tilde{t}_{R}$ mixing. The stop quarks of different handedness are related to the stop quark mass eigenstates $\tilde{t}_{+}, \tilde{t}_{-}$ through the following transformations:

$$
\begin{aligned}
& \tilde{t}_{L}=\cos \alpha_{t} \tilde{t}_{-}-e^{i \phi_{t}} \sin \alpha_{t} \tilde{t}_{+} \\
& \tilde{t}_{R}=e^{-i \phi_{t}} \sin \alpha_{t} \tilde{t}_{-}+\cos \alpha_{t} \tilde{t}_{+} .
\end{aligned}
$$

The only 1-loop diagram responsible for the generation of $\mathcal{A}_{b}$ is shown in Fig. 2. The reader can convince himself that there is no $\mathrm{CP}$-violating contributions coming from the $t-t$ self-energy diagram; it is again an illustration of the fact that rescattering into itself does not contribute to rate asymmetries. The $\tilde{b}_{L}-\tilde{b}_{R}$ mixing, which may also

Figure 2: The vertex correction to $\mathcal{A}_{b}$ in the SSM. The vertical dashed line denotes the absorptive part of the corresponding integral.

provide the necessary phase, has the same structure as the one for the top sector, with the substitutions: $\phi_{t} \rightarrow \phi_{b}$ and $\alpha_{t} \rightarrow \alpha_{b}$. However, if we assume that the scalar $b$-quarks are almost degenerate, their mixing effect can be neglected. The generalization is obvious. It is worthwhile to observe that, if we add phases $e^{i \phi_{\lambda}}$ and $e^{-i \phi_{\lambda}}$ to the terms $\left(\bar{\lambda}^{a} t_{L}\right)$ and $\left(\bar{\lambda}^{a} t_{R}\right)$ in Eq. (16), owing to the complex gluino mass, their effect can be absorbed into $\phi_{t}\left(\rightarrow \phi_{t}-2 \phi_{\lambda}\right)$ and $\phi_{b}\left(\rightarrow \phi_{b}-2 \phi_{\lambda}\right)$. Since the same interactions generate the neutron's electric dipole moment (NEDM), we have to take into account the limits originating from this measurement. However, direct restrictions on $\phi_{t / b}$ from the NEDM turn out not to be very reliable [16] and therefore will not be applied here. Indirect bounds may be obtained within the supergravity-induced SSM. However, as showed in Ref. [17], even assuming the same phase for all quark families, the model allows for maximal CP-violating phases for sufficiently heavy up- and down-squarks; therefore it is legitimate to assume maximal CP violation. It should be stressed here that we are not restricting ourselves to the minimal supergravity-induced models.

A very convenient way to parametrize the $t \rightarrow b W^{+}$and $\bar{t} \rightarrow \bar{b} W^{-}$decay vertices is the following:

$$
\begin{aligned}
\Gamma^{\mu} & =\frac{-i g V_{t b}^{K M}}{\sqrt{ } 2} \bar{u}\left(p_{b}\right)\left[\gamma^{\mu} P_{L}-\frac{2 p_{t}^{\mu}}{m_{W}} F_{2}^{R} P_{R}\right] u\left(p_{t}\right), \\
\bar{\Gamma}^{\mu} & =\frac{-i g V_{t b}^{K M^{*}}}{\sqrt{ } 2} \bar{v}\left(p_{\bar{t}}\right)\left[\gamma^{\mu} P_{L}+\frac{2 p_{\bar{t}}^{\mu}}{m_{W}} \bar{F}_{2}^{L} P_{L}\right] v\left(p_{\bar{b}}\right) .
\end{aligned}
$$

No other relevant form factor is generated at the 1-loop level of the perturbation expansion. We relate the rate asymmetry and the form factors as follows:

$$
\mathcal{A}_{b} \equiv \frac{\Gamma\left(t \rightarrow W^{+} b\right)-\Gamma\left(\bar{t} \rightarrow W^{-} \bar{b}\right)}{\Gamma\left(t \rightarrow W^{+} b\right)+\Gamma\left(\bar{t} \rightarrow W^{-} \bar{b}\right)}=\frac{\left(m_{t} / m_{W}\right) w^{2}\left(m_{t}^{2}, m_{b}^{2}, m_{W}^{2}\right) \operatorname{Re}\left(F_{2}^{R}-\bar{F}_{2}^{L}\right)}{2 m_{W}^{4}-m_{W}^{2} m_{t}^{2}-m_{W}^{2} m_{b}^{2}-\left(m_{t}^{2}-m_{b}^{2}\right)^{2}} .
$$


Here the kinematic function $w(x, y, z)=\left(x^{2}+y^{2}+z^{2}-2 x y-2 x z-2 z y\right)^{\frac{1}{2}}$. In the SSM, the $\mathrm{CP}$-violating factor, $\operatorname{Re}\left(F_{2}^{R}-\bar{F}_{2}^{L}\right)$, is

$$
\operatorname{Re}\left(F_{2}^{R}-\bar{F}_{2}^{L}\right)=-\frac{2 \alpha_{s}}{3 \pi} \sin \left(2 \alpha_{t}\right) \sin \left(\phi_{t}\right) \frac{m_{\lambda} m_{W}}{\left(m_{t}^{2}-m_{W}^{2}\right)^{2}} \operatorname{Im} \mathcal{I}\left(m_{\tilde{t}}^{2}\right)
$$

In this article, we assume that only the lightest stop quark $\tilde{t}_{+}$, of mass $m_{\tilde{t}}$, is available in the absorptive integral $\operatorname{Im} \mathcal{I}\left(m_{\tilde{t}}^{2}\right)$. We have

$$
\begin{gathered}
\operatorname{Im} \mathcal{I}\left(m_{\tilde{t}}^{2}\right) / \pi=\left(1+m_{W}^{2} / m_{t}^{2}\right) w\left(m_{t}^{2}, m_{\lambda}^{2}, m_{\tilde{t}}^{2}\right)-D L / w\left(m_{t}^{2}, m_{b}^{2}, m_{W}^{2}\right) ; \\
D=\left(m_{W}^{2}-m_{t}^{2}\right)^{2}-\left(m_{\tilde{t}}^{2}-m_{\lambda}^{2}-m_{t}^{2}\right)\left(m_{W}^{2}-m_{t}^{2}\right)-\left(m_{\tilde{b}}^{2}-m_{\lambda}^{2}\right)\left(m_{t}^{2}+m_{W}^{2}\right) \\
L=\log \left(L^{+} / L^{-}\right) ; \quad L^{ \pm}=F \pm w\left(m_{t}^{2}, m_{W}^{2}, m_{b}^{2}\right) w\left(m_{t}^{2}, m_{\tilde{t}}^{2}, m_{\lambda}^{2}\right) ; \\
F=2 m_{t}^{2}\left(m_{\lambda}^{2}+m_{b}^{2}-m_{\tilde{b}}^{2}\right)-\left(m_{t}^{2}-m_{\tilde{t}}^{2}+m_{\lambda}^{2}\right)\left(m_{t}^{2}+m_{b}^{2}-m_{W}^{2}\right) .
\end{gathered}
$$

In Fig. 3, we show a typical size of the rate asymmetry $\mathcal{A}_{b}$ at the level of one per cent,

Figure 3: The rate asymmetry $\mathcal{A}_{b}$ as a function of the scalar bottom quark mass $m_{\tilde{b}}$ for various $m_{t}$ when $m_{\lambda}=100 \mathrm{GeV}$ and $m_{\tilde{t}}=50 \mathrm{GeV}$.

for general cases. The best environment for measuring the rate asymmetry would mostly likely be the future linear $e^{+} e^{-}$collider at $500 \mathrm{GeV}$. Since it is expected that about 50000 $t \bar{t}$ events will be produced each year, the smallest possible measurable asymmetry will be of the order of $1 \%$, assuming that $B R\left(t \rightarrow W^{+} b\right) \simeq 50 \%$, for one year's run. Therefore we conclude that the measurement of the rate asymmetry induced within the SSM will be quite challenging. Noticing that if $t \bar{t}$ are pair produced, nonvansihing asymmetry must be an effect of $\mathrm{CP}$ violation in the process of $t \bar{t}$ decays because whenever $t$ is being produced there is an accompanied $\bar{t}$ and therefore the production mechanism cannot fake the asymmetry in the decay rates.

\section{CPT Constraints}

The CPT invariance guarantees that the particle and the antiparticle have the same total widths. Assuming no cancellation between different orders of perturbation expansion, we can therefore conclude that, order by order, contributions to the total widths should be the same. In particular this means that at the order of $\alpha^{2}$ :

$$
\sum_{\text {decay channels }} \Gamma(t \rightarrow \cdots)=\sum_{\text {decay channels }} \Gamma(\bar{t} \rightarrow \cdots) .
$$

One may have noticed that besides 2-body decays considered earlier there are also 3body decays contributing at this level of perturbation expansion. However their effect is

\footnotetext{
${ }^{3}$ Form factors defined in our previous paper [18] are related to those adopted here by the relations: $f_{2}^{L / R}=F_{2}^{L / R}, \bar{f}_{2}^{L / R}=\bar{F}_{2}^{L / R}$, etc. Therefore one can see that for CP-violating contributions we get $\left(F_{2}^{R}-\bar{F}_{2}^{L}\right)=\left(f_{2}^{R}-\bar{f}_{2}^{L}\right)=2 f_{2}^{R} C_{C V}$, where in the last step Eq.(3) of Ref. [18] has been used. It is clear how the CP-conserving pieces cancel.
} 
identical channel by channel, for $t$ and $\bar{t}$ and therefore irrelevant for our purpose. It is related to the fact that the $W$ self-energy amplitudes do not induce any rate asymmetry.

It is worthwhile to observe how the above constraint is fulfilled. Let us restrict ourself to the SM. In the case of self-energy induced asymmetries, it is particularly simple; one can see from the formula for the asymmetry, Eq. (9), that the rate difference summed over all 2-body final states (summation over $k$ ) could be written as:

$$
\begin{aligned}
& \sum_{k}\left[\Gamma\left(t \rightarrow W^{+} d_{k}\right)-\Gamma\left(\bar{t} \rightarrow W^{-} \bar{d}_{k}\right)\right] \\
\sim & \sum_{k} \int d \Phi\left(W d_{k}\right) \frac{1}{2}\left|\hat{A}_{1}\right|^{2} \sum_{i j} \operatorname{Im}\left(V_{t k}^{*} V_{t i} V_{j i}^{*} V_{j k}\right) \operatorname{Im}\left[\Sigma\left(m_{i}^{2}\right)\right] .
\end{aligned}
$$

An irrelevant factor from the $j$ propagator is not shown here. Since

$$
\int d \Phi\left(W^{+} d_{k}\right) \frac{1}{2}\left|\hat{A}_{1}\right|^{2}=m_{t} \operatorname{Im}\left[\Sigma\left(m_{k}^{2}\right)\right]
$$

we can write the rate difference summed over all 2-body final states as

$$
\sum_{k}\left[\Gamma\left(t \rightarrow W^{+} d_{k}\right)-\Gamma\left(\bar{t} \rightarrow W^{-} \bar{d}_{k}\right)\right] \sim \sum_{i j k} \operatorname{Im}\left(V_{t k}^{*} V_{t i} V_{j i}^{*} V_{j k}\right) \operatorname{Im}\left[\Sigma\left(m_{i}^{2}\right)\right] \operatorname{Im}\left[\Sigma\left(m_{k}^{2}\right)\right]=0 .
$$

It vanishes because $\operatorname{Im}\left(V_{t k}^{*} V_{t i} V_{j i}^{*} V_{j k}\right)$ is antisymmetric under $i \leftrightarrow k$ exchange. In the 2HDM, if $t \rightarrow H^{+} d_{k}$ is kinematically allowed one should include also this decay channel, the conclusion stays, of course, the same.

A similar situation occurs for the vertex-induced asymmetry within the SSM. Since we neglect any flavour mixing, there are only two 2-body decay channels opened: $t \rightarrow W^{+} b$ and $t \rightarrow \tilde{t} \lambda$. It is obvious from Eq. (23) that at least two decay channels must exist in order to generate a non-zero rate asymmetry. Equation (23) tells us also that the following relation is satisfied:

$$
\left[\Gamma\left(t \rightarrow W^{+} b\right)-\Gamma\left(\bar{t} \rightarrow W^{-} \bar{b}\right)\right]=-\left[\Gamma(t \rightarrow \tilde{t} \lambda)-\Gamma\left(\bar{t} \rightarrow \tilde{t}^{\dagger} \bar{\lambda}\right)\right] .
$$

It is convenient to adopt here a phase convention in which $\mathrm{CP}$ violation in the process $t \rightarrow W^{+} b$ appears only in the $\left(t \tilde{t}_{+} \lambda\right)$ vertex. In such basis it is easy to separate out $\mathrm{CP}$-violating phases in the tree-level amplitude $A_{1}(t \rightarrow \tilde{t} \lambda)$ and the 1-loop amplitude $A_{2}\left(t \rightarrow W^{+} b\right)$. It will be convenient to adopt the following definitions for CP-violating contributions to those amplitudes:

$$
A_{2}\left(t \rightarrow W^{+} b\right)=e^{i \phi} \hat{A}_{2}\left(t \rightarrow W^{+} b\right), \quad A_{1}(t \rightarrow \tilde{t} \lambda)=e^{i \phi} \hat{A}_{1}(t \rightarrow \tilde{t} \lambda) .
$$

In order to see how the CPT-constraint is satisfied, it is instructive to write down an expression for the asymmetry before the loop integration is performed, by means of the Cutkosky [19 rule for the appropriate absorptive part:

$$
\begin{aligned}
& {\left[\Gamma\left(t \rightarrow W^{+} b\right)-\Gamma\left(\bar{t} \rightarrow W^{-} \bar{b}\right)\right] \sim} \\
& \quad-2 \operatorname{Im}\left(e^{i \phi}\right) \int d \Phi(\tilde{t} \lambda) \int d \Phi\left(W^{+} b\right) \hat{A}_{1}(t \rightarrow \tilde{t} \lambda) A(W b \rightarrow \tilde{t} \lambda)^{*} A\left(t \rightarrow W^{+} d_{k}\right)^{*} .
\end{aligned}
$$

For formal reasons we put stars to indicate complex conjugations, although both $A(W b \rightarrow$ $\tilde{t} \lambda)$ and $A\left(t \rightarrow W^{+} d_{k}\right)$ are real numbers; $\int d \Phi(\cdots)$ represents an appropriate phase-space integration including summation over spins of on-shell particles. The reader can easily verify that an analogous rate difference for $t \rightarrow \tilde{t} \lambda$ would have an opposite sign. 


\section{Summary}

We have discussed the $\mathrm{CP}$ violation in the decay rate-asymmetry of the top quark in various models. We found effect at a level of a per cent in the SSM, where a CP-violating phase may occur in the $\tilde{t}_{L}-\tilde{t}_{R}$ mixing. We also illustrate how the CPT constraint manifests itself in explicit calculations such that the total widths of a top quark and an antitop quark are equal.

\section{Acknowledgments}

We thank J. Liu for a useful discussion. We are grateful to the CERN Theory Division for the support during this work. This research was also supported in part by the U.S. Department of Energy.

\section{References}

[1] I. Bigi, Y. Dokshitzer, V. Khoze, J. Kühn and P.M. Zerwas, Phys. Lett. 181B (1986) 157.

[2] T.D. Lee, Phys. Rev. D8 (1973) 1226;

S. Weinberg, Phys. Rev. D42 (1990) 860.

[3] K. Kobayashi and T. Maskawa, Prog. Theor. Phys. 49 (1973) 652.

[4] M. Bander, D. Silverman and A. Soni, Phys. Rev. Lett. 43 (1979) 242.

[5] J.-M. Gérard and W.-S. Hou, Phys. Rev. Lett. 62 (1989) 855;

L. Wolfenstein, Phys. Rev. D43 (1991) 151;

J.-M. Gérard and W.-S. Hou, Phys. Rev. D43 (1991) 2909;

H. Simma, G. Eilam and D. Wyler, Nucl. Phys. B352 (1991) 367.

[6] G. Eilam, J.L. Hewett and A. Soni, Phys. Rev. Lett. 67 (1991) 1979;

J.M. Soares, Phys. Rev. Lett. 68 (1992) 2102;

B. Grządkowski and J.F. Gunion, Phys. Lett. 289B (1992) 440;

D. Atwood et al., Phys. Rev. Lett. 70 (1993) 1364;

J. Liu, Phys. Rev. D48 (1993) 212;

D. Atwood et al., Brookhaven preprint TECHNION-PH-92-39, BNL-48161 (1992);

D. Atwood, G. Eilam and A. Soni, Stanford preprint SLAC-PUB-6083 (1993).

[7] J.F. Donoghue and G. Valencia, Phys. Rev. Lett. 58 (1987) 451;

F. Hoogeveen and L. Stodolski, Phys. Lett. 212B (1988) 505;

W. Bernreuther and O. Nachtmann, Phys. Rev. Lett. 63 (1989) 2787;

W. Bernreuther, U. Löw, J.P. Ma and O. Nachtmann, Z. Phys. C43 (1989) 117;

C.A. Nelson, Phys. Rev. D41 (1990) 2805;

G. Valencia and A. Soni, Phys. Lett. 263B (1991) 517;

J. Körner, J.P. Ma, R.Münch, O. Nachtmann and R. Schöpf, Z. Phys. C49 (1991) 447 ; 
W. Bernreuther, G.W. Botz, O. Nachtmann and P. Overmann, Z. Phys. C52 (1991) 567 ;

W. Bernreuther, J.P. Ma and T. Schröder, Phys. Lett. 297B (1992) 318;

D. Atwood and A. Soni, Phys. Rev. D45 (1992) 2405;

D. Atwood, A. Aeppli and A. Soni, Phys. Rev. Lett. 69 (1992) 2754;

A. Aeppli and A. Soni, Phys. Rev. D46 (1992) 315;

A. Brandenburg and J.P. Ma, Phys. Lett. 298B (1993) 211.

[8] W. Bernreuther, O. Nachtmann, O. Overmann and T. Schröder, Nucl. Phys. B388 (1992) 53;

W. Bernreuther, T. Schröder and T.N. Pham, Phys. Lett. 279B (1992) 389.

[9] B. Grządkowski and J.F. Gunion, Phys. Lett. 287B (1992) 237;

B. Grządkowski and J.F. Gunion, Phys. Lett. 294B (1992) 361;

G.L. Kane, G.A. Ladinsky and C.-P. Yuan, Phys. Rev. D45 (1992) 124;

R.H. Dalitz and G.R. Goldstein, Phys. Rev. D45 (1992) 1531;

C.R. Schmidt and M.E. Peskin, Phys. Rev. Lett. 69 (1992) 410;

D. Chang and W.-Y. Keung, Phys. Lett. 305B (1993) 261;

D. Chang, W.-Y. Keung and I. Phillips, CERN preprint CERN-TH.6814/93, Phys. Rev. D (in press);

A. Soni and R.M. Xu, Phys. Rev. Lett. 69 (1992) 33;

A. Pilaftsis and M. Nowakowski, Dortmund preprint DO-TH/92-27;

J.P. Ma and B.H.J. McKellar, University of Melbourne preprint UM-P-93/16.

[10] B. Grządkowski, Phys. Lett. 305B (1993) 384;

D. Chang, W.-Y. Keung and I. Phillips, CERN preprint CERN-TH.6658/92, Nucl. Phys. B (in press).

[11] W. Bernreuther and P. Overmann, Heidelberg preprint HD-THEP-93-11.

[12] L.-L. Chau and W.-Y. Keung, Phys. Rev. Lett. 53 (1984) 1802;

C. Jarlskog and R. Stora, Phys. Lett. 208B (1988) 268.

[13] L. Wolfenstein, Phys. Rev. Lett. 51 (1984) 1945.

[14] G. Isidori, Phys. Lett. 298B (1993) 409.

[15] C.R. Schmidt, Phys. Lett. 293B (1992) 111;

C.J.-C. Im, G.L. Kane and P.J. Malde, Michigan preprint UM-TH-92-27;

R. Garisto and G. Kane, TRIUMF preprint TRI-PP-1, January 1993.

[16] A. De Rújula, M.B. Gavela, O. Pène and F.J. Vegas, Phys. Lett. 245B (1990) 640;

D. Chang, W.-Y. Keung, and T.C. Yuan, Phys. Lett. 251B (1990) 608;

J.F. Gunion and R. Vega, Phys. Lett. 251B (1990) 21.

[17] Y. Kizukuri and N. Oshimo, Phys. Rev. D46 (1992) 3025.

[18] B. Grzạdkowski and W.-Y. Keung, CERN-TH.6917/93 (1993), Phys. Lett. B (in press).

[19] R.E. Cutkosky, J. Math. Phys. 1 (1960) 429. 